\title{
Analysis of Pulmonary Nodule Evolutions Using a Sequence of Three-Dimensional Thoracic CT Images
}

\author{
Y. Kawata ${ }^{1}$, N. Niki ${ }^{1}$, H. Ohmatsu ${ }^{2}$, M. Kusumoto ${ }^{3}$, R. Kakinuma ${ }^{2}$,

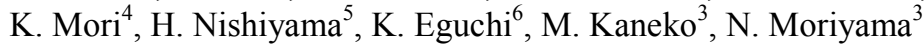 \\ ${ }^{1}$ Dept. of Optical Science, Univ. of Tokushima, \\ \{kawata, niki\}@opt.tokushima-u.ac.jp \\ ${ }^{2}$ National Cancer Center East, \\ ${ }^{3}$ National Cancer Center, ${ }^{4}$ Tochigi Cancer Center, \\ ${ }^{5}$ The Social Health Medical Center, ${ }^{6}$ National Shikoku Cancer Center
}

\begin{abstract}
This paper presents a method to analyze volume evolutions of pulmonary nodules for discrimination between malignant and benign nodules. Our method consists of four steps; (1) The 3-D rigid registration of the two successive 3-D thoracic CT images, (2) the 3-D affine registration of the two successive region-of-interest (ROI) images, (3) non-rigid registration between local volumetric ROIs, and (4) analysis of the local displacement field between successive temporal images In preliminary study, the method was applied to the successive 3-D thoracic images of two pulmonary lesions including a metastasis malignant case and a inflammatory benign to quantify the evolving process in the pulmonary nodules and surrounding structure. The time intervals between successive 3-D thoracic images for the benign and malignant cases were 150 and 30 days, respectively. From the display of the displacement fields and the contrasted image by the vector field operator based on the Jacobian, it was observed that the benign case reduced in the volume and the surrounding structure was involved into the nodule in the evolution process. It was also observed that the malignant case expanded in the volume. These experimental results indicate that our method is a promising tool to quantify how the lesions evolve their volume and surrounding structures.
\end{abstract}

\section{Introduction}

Physicians often wish to compare a sequence of thoracic CT images of the same patient and to analyze dynamical characteristic of pulmonary nodules. Computerized differentiation between the thoracic CT image sequences may assist physicians to detect abnormalities and to characterize interval changes in the known lung lesions. Though the correspondence between slice images of the nodule over time is interactively performed, the promising results for evaluating the likelihood of malignancy have been presented by using the sequence of thoracic CT images. Swensen et al [1] presented a technique based on differential enhancement of pulmonary nodules after the intravenous administration of iodinated contrast material to evaluate the likelihood of malignancy. Other group also demonstrated that the nodule enhancement was an indicator of malignancy based on the succession of 
thoracic CT images [2]. Yankeleviz et al. presented a technique to evaluate nodule growth based on sequential thin-section CT images [3]. In their technique the nodule growth was assessed by comparison the image from the initial scan including the maximal area with the image form the repeat scan including the maximal area. This comparison was done by displaying the two image sets side by side. However, the thoracic CT images are not usually reproducible in terms of patient positioning, inspiration, and cardiac pulsation. The complexity of the lung deformation makes the analysis of sequential thoracic CT images difficult. In comparison with the existing approaches of deformation analysis of brain and heart diseases, few works have been done to address the problem of tracking interval changes in thoracic CT image sequences [4]. Fan and Chen [5] presented an approach to estimate volumetric lung warping and registration from 3-D CT images obtained at different stages of breathing. Their warping model was governed by a model derived from continuum mechanics and a 3-D optical flow.

The purpose of this study is to aid differential diagnosis of lung lesions through quantitative analysis of interval changes of the 3-D pulmonary nodule with respect to the internal structure of the nodule, the nodule margin characteristics, and the relationship between surroundings and the nodule. In this paper, we present an approach to analyze the displacement field of thoracic CT image sequences combining with 3-D rigid and affine registration, non-rigid registration, and vector field analysis. We apply our method to sequences of 3-D thoracic images with lesions to evaluate interval changes of 3-D pulmonary nodules.

\section{Methods}

\subsection{Overview}

The method consists of four steps; 1) The 3-D rigid registration of the two successive 3-D thoracic CT images., 2) the 3-D affine registration of the two successive regionof-interest (ROI) images extracted from two registered 3-D thoracic CT images, 3) non-rigid registration between local volumetric ROIs, 4) analysis of the local displacement field between successive temporal images. For the first step, we computed a rigid transformation between two successive 3-D thoracic CT images $I_{1}$ and $I_{2}$. We then resampled the image $I_{2}$ into $I_{2}$ ' to superpose $I_{2}$ to $I_{1}$ roughly. To avoid complexity of the whole lung deformation caused by a combination of body movement, heartbeats, and respiration, we interactively selected and extracted a local ROI image including a nodule of interest from the registered 3-D thoracic CT image. The ROI image was a cubic region $\left(128 \times 128 \times 128\right.$ voxels). For both $I_{1}$ and $I_{2}$ ' the same location of the nodule center was interactively determined and the cubic region centering on this location was extracted. The ROI images extracted from $I_{1}$ and $I_{2}$ ' are denoted as $F_{1}$ and $F_{2}$, respectively. We then computed an affine transformation between two ROI images, $F_{1}$ and $F_{2}$, and we resampled image $F_{2}$ into $F_{2}$ ' to superpose to $F_{1}$. In our experiments we always resample the data into isotropic volumes before processing them. As previously ascertained by other researches [6],[7], the mutual information confirms to be a successful measure of goodness of fit for registration of images. Studholme presented that mutual information is not 


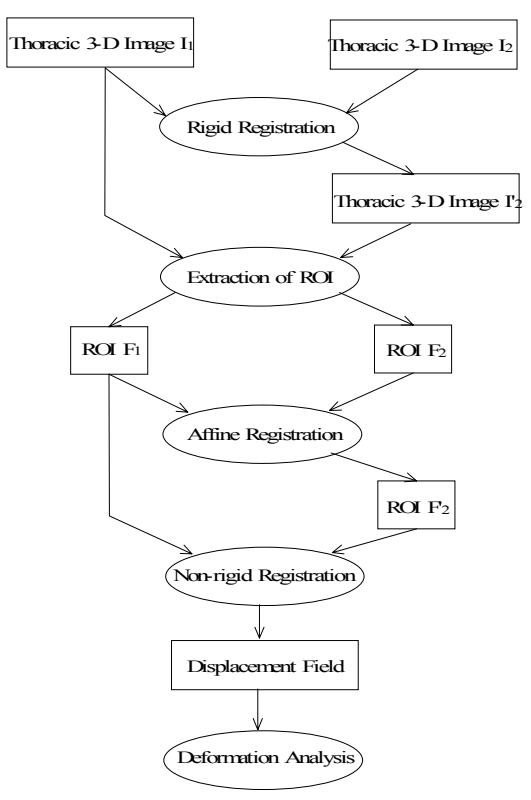

Fig. 1. Block diagram of the method.

independent of the overlap between two images and suggested the use of the normalized mutual information as a measure of image alignment [8]. We used the normalized mutual information as a voxel similarity measure. Next, we computed the non-rigid displacement between $F_{1}$ and $F_{2}$ ' using a warping function which maps $F_{1}$ to $F_{2}$ '. The warping function is a displacement field represented by a 3-D array of displacement vectors. To design the warping function, we used the active net model proposed by Sakaue [9] for the extraction of a texture region. The active net model is a two-dimensional (2-D) elastic network model with is a 2-D extension of Snakes proposed by Kass [10]. This model deforms the net to wrap a target region minimizing the internal strain energy of the net and the image energy that attracts the net toward features such as edge of the target region. Witkin et al. formulated multidimensional matching and registration problems as the minimization of an energy measure that integrates a similarity term and a smoothness term of the deformation field [11]. Following their idea, the active net model also might be applied to estimate the non-rigid displacement by replacing the image energy with the integral of the density difference between the image sequences. In this study, we extended the active net into 3-D, which is called 3-D active net to estimate a volume displacement field between $F_{1}$ and $F_{2}$ '. After computing the local displacement field between successive ROI images, we analyzed the displacement filed using the vector field operator based on the Jacobian operator [13]. 


\subsection{Non-rigid Registration}

\subsubsection{Warping Function}

We compute the 3-D displacement field with a non-rigid transformation based on a warping function which transforms $F_{1}$ to $F_{2}$, which represents as the affine transformation result of $F_{2}$. The warping function is designed by introducing a 3-D active net model which minimizes the energy functional and deforms elastically [9]. The energy functional consists of the image energy and the internal strain energy of the net. The image energy was obtained by the integral of the density difference of each voxels between $F_{1}$ and $F_{2}$ '. The internal strain energy of the 3-D net constrains the non-rigid transformation to be smooth assuming that the local displacement field of the nodules is smooth. This term plays a role to shrink and smooth the 3-D net. By using three parameters, $p, q$, and $r$, points on the 3-D nets for 3-D images $F_{1}$ and $F_{2}$, are defined by $\mathbf{w}(p, q, r)=(x(p, q, r), y(p, q, r), z(p, q, r))$ and $\mathbf{v}(p, q, r)=(X(p, q, r), Y(p, q, r)$, $Z(p, q, r))(0 \leq p \leq 1,0 \leq q \leq 1,0 \leq r \leq 1)$, respectively. The warping function is given by a 3-D displacement field that derived from vectors $\mathbf{w}$. The 3-D displacement field is obtained by minimizing the energy functional, while the position of each vector $\mathbf{v}$ is fixed. The image energy term of 3-D active net is defined as

$$
S(\mathbf{w})=\int_{0}^{1} \int_{0}^{1} \int_{0}^{1}\left(F_{2}^{\prime}(\mathbf{v}(p, q, r))-F_{1}(\mathbf{w}(p, q, r))\right)^{2} d p d q d r
$$

The internal strain energy of the 3-D active net is given by

$$
\begin{aligned}
R(\mathbf{w})= & \int_{0}^{1} \int_{0}^{1} \int_{0}^{1} \frac{\alpha}{2}\left[\left(\frac{\partial \mathbf{w}}{\partial p}\right)^{2}+\left(\frac{\partial \mathbf{w}}{\partial q}\right)^{2}+\left(\frac{\partial \mathbf{w}}{\partial r}\right)^{2}\right]+ \\
& \frac{\beta}{2}\left[\left(\frac{\partial^{2} \mathbf{w}}{\partial p^{2}}\right)^{2}+\left(\frac{\partial^{2} \mathbf{w}}{\partial q^{2}}\right)^{2}+\left(\frac{\partial^{2} \mathbf{w}}{\partial_{r}^{2}}\right)^{2}+2\left(\frac{\partial^{2} \mathbf{w}}{\partial p \partial q}\right)^{2}+2\left(\frac{\partial^{2} \mathbf{w}}{\partial q \partial r}\right)^{2}+2\left(\frac{\partial^{2} \mathbf{w}}{\partial_{r} \partial p}\right)^{2}\right] d p d q d r
\end{aligned}
$$

where $\alpha$ and $\beta$ are weighting parameters to control the first and the second terms. The first term results in the force to shrink the 3-D net. The second term makes the 3-D net shape smooth. Associated with the smoothness of the transformation $R$, the following energy functional of the 3-D active net is obtained.

$$
\begin{aligned}
J(\mathbf{w})=\int_{0}^{1} \int_{0}^{1} \int_{0}^{1}\left(F_{2}^{\prime}(\mathbf{v}(p, q, r))-F_{1}(\mathbf{w}(p, q, r))\right)^{2} \\
+\frac{\alpha}{2}\left[\left(\frac{\partial \mathbf{w}}{\partial p}\right)^{2}+\left(\frac{\partial \mathbf{w}}{\partial q}\right)^{2}+\left(\frac{\partial \mathbf{w}}{\partial r}\right)^{2}\right] \\
+\frac{\beta}{2}\left[\left(\frac{\partial^{2} \mathbf{w}}{\partial p^{2}}\right)^{2}+\left(\frac{\partial^{2} \mathbf{w}}{\partial q^{2}}\right)^{2}+\left(\frac{\partial^{2} \mathbf{w}}{\partial r^{2}}\right)^{2}+2\left(\frac{\partial^{2} \mathbf{w}}{\partial p \partial q}\right)^{2}+2\left(\frac{\partial^{2} \mathbf{w}}{\partial q \partial r}\right)^{2}+2\left(\frac{\partial^{2} \mathbf{w}}{\partial_{r} \partial p}\right)^{2}\right] d p d q d r
\end{aligned}
$$




\subsubsection{Implementation}

As a practical computation, the 3-D net is approximated by a $n \times m \times l$ mesh;

$$
\mathbf{w}(p, q, r)=\mathbf{w}(i \Delta n, j \Delta m, k \Delta l)=\left(x_{i, j, k}, y_{i, j, k}, z_{i, j, k}\right), 0 \leq i \leq n, 0 \leq j \leq m, 0 \leq k \leq l
$$

where $\Delta n=1 / n, \Delta m=1 / m$, and $\Delta l=1 / l$. The grid points are placed on the cubic mesh. Each grid of 3-D net has 26-neighbors. The first term in Eq. (2) results in the force to shrink the boundary of the 3-D net. To prevent the boundary gird from shrinking, we fixed the position of the boundary nod of the 3-D net. We used an iterative technique [9] to obtain the 3-D active net that minimizing the energy functional in Eq. (3). The ROI images that we intend to match often contain vessels or bronchi with smaller diameter size than that of the nodule of interest. Therefore, we incorporated a hierarchical multiresolution approach in which the resolution of the mesh is increased, along with the image resolution in a coarse to fine manner.

\subsection{Vector Field Operator}

We characterize the obtained displacement field by using a vector field operator that transforms a 3-D vector field into 3-D scalar image. The value of each voxel in the scalar image varies with respect to the nodule evolution. Some vector field operators of the displacement field have been proposed. Thirion et al. proposed vector field operations based on the divergence and the norm of the displacement field [12]. Rey et al. pointed out that values obtained by their operators were hardly interpreted in terms of physical meanings and proposed another operator based on the Jacobian of the warping function that was derived from the displacement field [13]. They also demonstrated that the vector field operator based on the Jacobian provides a measure of evolution of a small volume. In this study, we adopt the Jacobian operator proposed by Rey. Let the value of the Jacobian operator at the point $P$ be $J$. When $J>1$ , the evolution at the point $P$ is considered as a local expansion and when $J<1$, the evolution at the point $P$ is considered as a local shrinking. When $\mathrm{J}=1$, no variation of volume at the point $P$ is observed. The computation of the first derivatives in the Jacobian of the warping function was performed by using the recursive filtering proposed by Derich [14].

\section{Results and Discussion}

Thin-section CT images were obtained by the helical CT scanner (Toshiba TCT900S Superhelix) under the following conditions; beam width: $2 \mathrm{~mm}$, table speed: $2 \mathrm{~mm} / \mathrm{sec}$, tube voltage; $120 \mathrm{kV}$, tube current: $250 \mathrm{~mA}$. For the scan duration, patients held their breath at full inspiration. Per patient, about 60 slices at $1 \mathrm{~mm}$ intervals were obtained to observe the whole nodule region and its surroundings. The range of pixel size in each square slice of 512 pixels was between $0.3 \times 0.3 \mathrm{~mm}^{2}$ and $0.4 \times 0.4 \mathrm{~mm}^{2}$. The $3-\mathrm{D}$ thoracic image was reconstructed by a linear interpolation technique to make each voxel isotropic.

We present the application results of our method to two sets of successive 3-D thoracic CT images $A$ and $B$. The data set $A$ contains sequential 3-D thoracic CT 


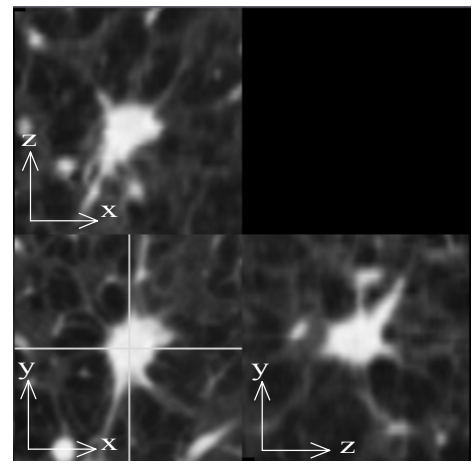

(a)

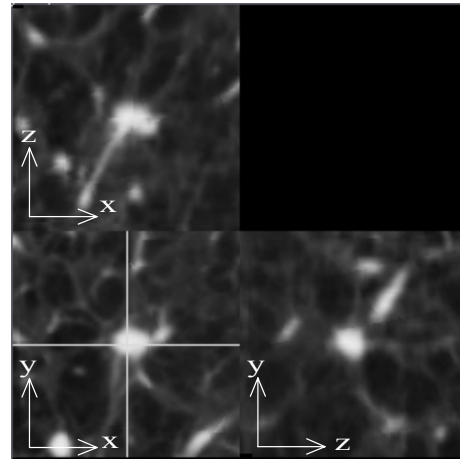

(b)

Fig. 2. ROI images of the benign nodule. (a) ROI image $C_{0}$ measured at $T_{0}$. (b) ROI image $C_{1}$ measured at $T_{1}$. Cross-sections for the xy-plane, yz-plane, xz-plane are shown.

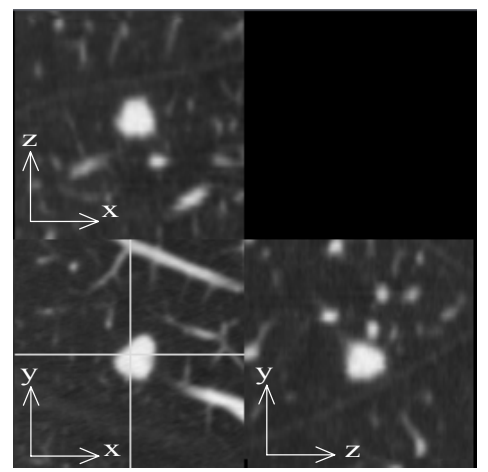

(a)

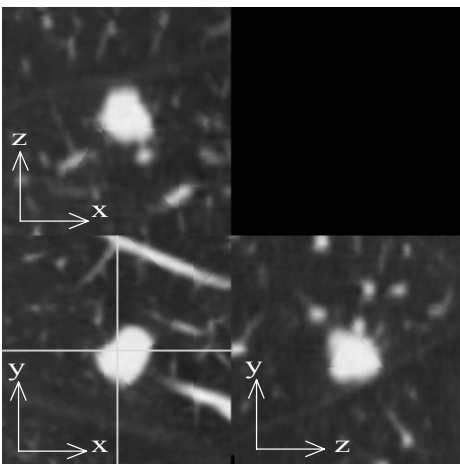

(b)

Fig.3. ROI images of the malignant nodule. (a) ROI image $D_{0}$ measured at $T_{2}$. (b) ROI image $D_{1}$ measured at $T_{3}$. Cross-sections for the xy-plane, yz-plane, xz-plane are shown.

images $A_{0}$ and $A_{1}$ of a patient with a benign nodule. These images $A_{0}$ and $A_{1}$ measured at different time $T_{0}$ and $T_{1}$, respectively. The period between $T_{0}$ and $T_{1}$ was 150 days. The data set $B$ contains sequential 3-D thoracic CT images $B_{0}$ and $B_{1}$ of a patient with a malignant nodule. These images $B_{0}$ and $B_{1}$ measured at different time $T_{2}$ and $T_{3}$, respectively. The period between $T_{2}$ and $T_{3}$ was 30 days.

Figs. 2 and 3 show ROI images of benign $\left(A_{0}\right.$ and $\left.A_{1}\right)$ and malignant $\left(B_{0}\right.$ and $\left.B_{1}\right)$ It is observed that the benign nodule reduces its volume over time and the malignant nodule expands its volume over time.

We computed the displacement fields between two consecutive ROI images by using the non-rigid registration. Fig. 4 shows the deformed grids of the benign and malignant nodules. It can be observed that the effect of a shrinking and an expansion of the grids for benign and malignant nodules evolutions. Fig.5 shows application 


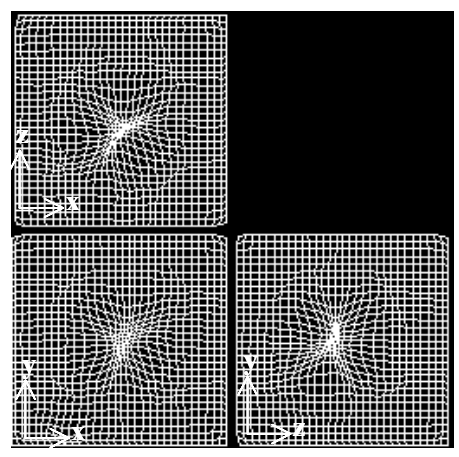

(a)

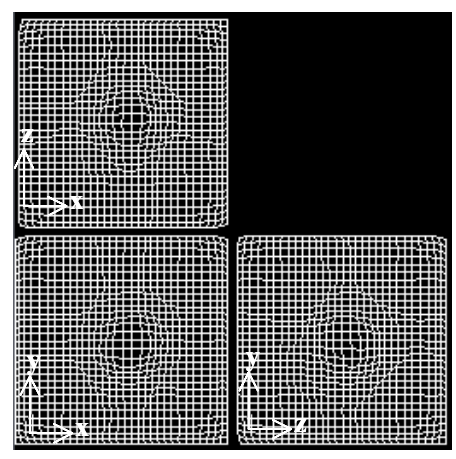

(b)

Fig. 4. 3-D Displacement fields. (a) Benign nodule. (b) Malignant nodule. Cross-sections of 3-D displacement field for the xy-plane, yz-plane, xz-plane are shown.

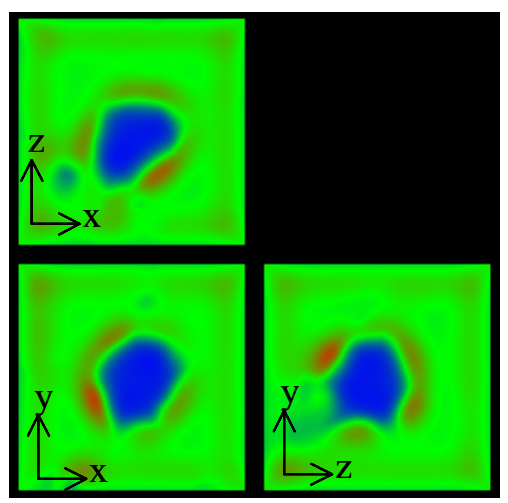

(a)

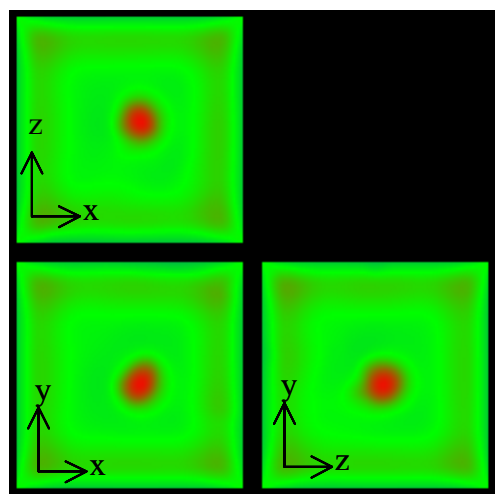

(b)

Fig.5. Applications of the vector field operator based on the Jacobian. (a) Benign nodule. (b) Malignant nodule. Cross-sections of 3-D displacement field for the xy-plane, yz-plane, xz-plane are shown.

results of the vector field operator based on the Jacobian. The more contrasted areas demonstrate the shrinking or the growing regions of nodules. From the display of the displacement fields and the contrasted image by the Jacobian operator, it is observed that the benign case reduces in the volume and the surrounding structure is involved into the nodule in the evolution process. It is also observed that the malignant case expands in the volume without deform the surrounding structure.

The application results of our method to the sequence of 3-D thoracic images demonstrate that the time interval changes of lesions can be made visible. Additionally, the experimental results shows that the displacement field computed by our method might be used to quantify how the lesions evolve their volume and surrounding structures by using the vector field operator based on the Jacobian. Real pulmonary nodules have a complex evolving process types such as tissue 
deformation, tissue displacement into other tissues, and combination of them. Still, the quantitative analysis of the displacement field of consecutive nodule images could aid physician diagnosis of indeterminate pulmonary lesions using only the static 3-D thoracic image.

\section{Conclusion}

We have presented a volumetric analysis method in evolving processes of pulmonary nodules by combining with 3-D rigid and affine registration, non-rigid registration, and vector field analysis. The application results of our method to the sequence of 3$\mathrm{D}$ thoracic images have demonstrated that the time interval changes of lesions can be made visible. Additionally we have presented that the deformation field and the contrasted image by the vector field operator might be used to quantify how the lesions evolve their volume and surrounding structures. To analyze the capabilities of our method, we are collecting successive 3-D thoracic CT images from appropriate clinical cases.

\section{References}

1. S. J. Swensen, R. L. Morin, B. A. Schueler, L. R. Brown, D. A. Cortese, P. C. Pairolero, W. M. Brutinel : Solitary pulmonary nodule: CT evaluation of enhancement with iodinated contrast material - A preliminary report. Radiology, 182, (1992) 343-347

2. K. Yamashita, S. Matsunobe, T. Tsuda, T. Nemoto, K. Matsumoto, H. Miki, J. Konishi, : Solitary pulmonary nodule: Preliminary study of evaluation with incremental dynamic CT. Radiology, 194, (1995) 399-405

3. D. F. Yankelevitz, R. Gupta, B. Zhao, C. I. Henschke : Small pulmonary nodules: Evaluation with repeat CT - Preliminary experience. Radiology, 212, (1999) 561-566

4. J. B. A. Maintz and M. A. Viergever: A survey of medical image registration. Medical Image Analysis, 2, (1998) 1-36

5. L. Fan, C. W. Chen: 3D warping and registration from lung images. Proc. SPIE, 3660, (1999) 459-470

6. F. Maes, A. Collignon, D. Vandermeulen, G. Marchal, and P. Suetens: Multimodality image registration by maximization of mutual information. IEEE Trans. Medical Imaging, 16, (1997) $187-198$

7. P. Viola, W. M. Wells III : Alignment by maximization of mutual information. Int. J. Computer Vision, 24, (1997) 137-154

8. C. Studholme, D. L. G. Hill and D. J. Hawkes: An overlap invariant entropy measure of 3D medical image alignment. Pattern Recognition, 32, (1998) 71-86

9. K. Sakaue and K. Yamamoto : Active net model and its application to region extraction. Journal of the Institute of Television Engineering, 45, (1991) 1155-1163

10. M. Kass, A. Witkin and D. Terzopulos : Snakes: Active contour models. International Journal of Computer Vision, 1, (1988) 321-331

11. A. Witkin, D. Terzopoulos, A.M. Kass : Signal matching through scale space, International Journal of Computer Vision, 1, (1987), 133-144.

12. J.-P. Thirion and G. Calmon: Deformation analysis to detect and quantify active lesions in three-dimensional medical image sequences. IEEE Trans. Medical Imaging, 18, (1999) 429441

13. D. Rey, G. Subsol, H. Delingette, and N. Ayache : Automatic detection and segmentation of evolving processes in 3D medical images: Application to multiple sclerosis. Proc. Information Processing in Medical Imaging, IPMI'99, (1999) 154-167

14. R. Deriche : Recursively implementing the gaussian and its derivatives. INRIA Research Report, 1893, (1993) 